msh-mss Mathématiques et sciences humaines

160 | Hiver 2002

Varia

\title{
Claude Berge, ses graphes et hypergraphes
}

Claude Berge, his graphs and hypergraphs

\section{Pierre Rosenstiehl}

\section{OpenEdition}

Journals

Édition électronique

URL : http://journals.openedition.org/msh/2915

DOI : $10.4000 /$ msh.2915

ISSN : 1950-6821

\section{Éditeur}

Centre d'analyse et de mathématique sociales de l'EHESS

\section{Édition imprimée}

Date de publication : 1 décembre 2002

ISSN : 0987-6936

\section{Référence électronique}

Pierre Rosenstiehl, « Claude Berge, ses graphes et hypergraphes », Mathématiques et sciences humaines [En ligne], 160 | Hiver 2002, mis en ligne le 10 février 2006, consulté le 23 juillet 2020. URL : http://journals.openedition.org/msh/2915 ; DOI : https://doi.org/10.4000/msh.2915 
Math. \& Sci. hum., (40 année, $\mathrm{n}^{\circ} 160,2002$, p. 7-12)

\section{Hommage du C.A.M.S. à Claude Berge}

\section{CLAUDE BERGE, SES GRAPHES ET HYPERGRAPHES}

Mathématicien, combinatoricien, sculpteur, anthropologue amateur, grand voyageur, collectionneur d'art indonésien, oulipien, joueur d'échecs et de pions exotiques, auteur de manuels, directeur de recherche renommé pour ses conférences de 30 minutes, compagnon de 35 ans du Centre de Mathématique Sociale, chercheurCNRS émérite affecté à l'EHESS jusqu'au 30 juin 2002, tel était Claude Berge.

Ainsi Vasek Chvátal de Rudgers University écrit : "The departure of Claude Berge leaves a painful void in many of our lives. He enchanted people around him with his multiple talents, his great erudition in diverses domains, his sense of humour, his modesty, his love of life ».

Amoureux des beaux énoncés, des phrases sibyllines, des problèmes insolubles et des démonstrations courtes, Berge préfère les conjectures aux théorèmes. Sa conjecture des «graphes parfaits » lui vaut une renommée mondiale : elle a en effet résisté aux meilleurs mathématiciens pendant quarante-deux ans. Il se régalait de voir publications, séminaires, conférences, colloques, tourner en vain autour de sa conjecture. Plus dura l'énigme, plus il était content : un labyrinthe se tissait, l'énigme portait en puissance de nombreuses propositions, elles-mêmes sources de nouvelles interrogations. Nous en donnons ci-dessous un énoncé " popularisé », c'est-à-dire accessible à tout un chacun (ce texte a été écrit pour la lecture des Jeudis de l'Oulipo du 7 novembre 2002 à l'Université Paris VII. Claude Berge est avec Raymond Queneau et François Le Lionnais membre fondateur de l'Ouvroir de littérature potentielle).

\section{DÉBUT ET FIN DE LA CONJECTURE DES GRAPHES PARFAITS : CECI EST UN TROU}

Il faut imaginer dans sa tête des trucs qu'on appelle sommets, et pour toute paire de sommets soit une arête qui les joint, soit une non-arête qui les laisse sans joint : ceci est un graphe selon Berge.

Il faut imaginer que l'on sait sauter de sommet en sommet par des arêtes, toujours nouvelles, en revenant au sommet de départ. Si les sommets rencontrés n'ont pas d'autres arêtes entre eux : ceci est un trou. Trou impair lorsque le nombre de sauts est impair (voir dessin dans la tête). 


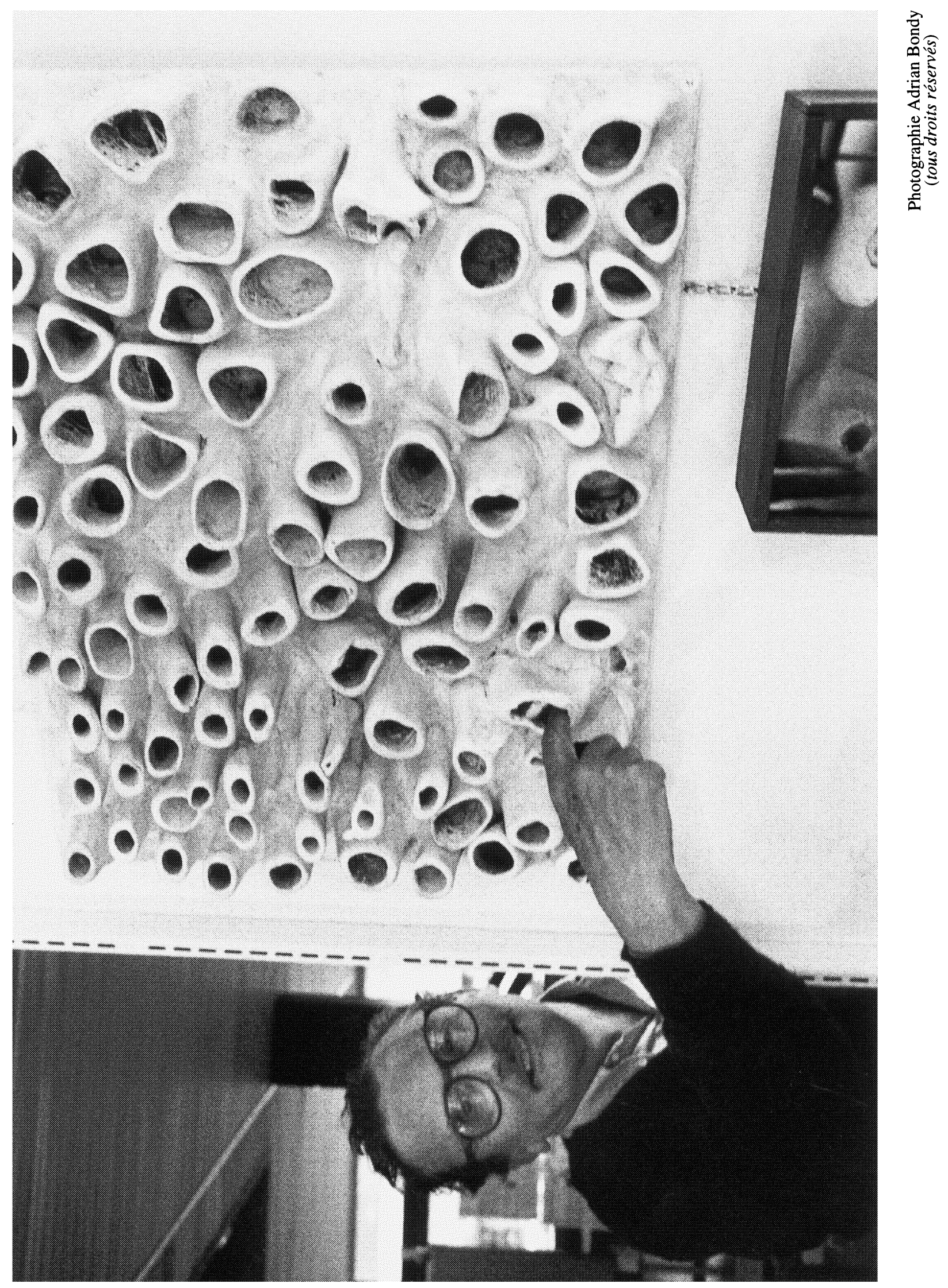


Il faut imaginer que l'on sait sauter de sommet en sommet par des non-arêtes, toujours nouvelles, en revenant au sommet de départ. Si les sommets rencontrés n'ont pas d'autres non-arêtes entre eux : ceci est un antitrou (voir dessin dans la tête).

Et parfait ?

Il faut imaginer pour cela un peintre économe qui donne une couleur à chaque sommet du graphe, deux sommets joints par une arête devant toujours être de couleurs différentes. Il minimise le nombre de couleurs : ceci est le nombre chromatique du graphe.

Il faut imaginer un sociologue mafieux qui cherche des cliques, c'est-à-dire des groupes de sommets tous joints deux à deux par une arête. Il maximise la taille de la clique : ceci est le nombre cliquetique du graphe.

Il faut alors imaginer un graphe tel que, pour lui et chacun de ses sous-graphes, les nombres chromatiques et cliquetiques sont égaux : ceci est un graphe parfait. (Par sousgraphe on entend un sous-ensemble des sommets joints par les arêtes correspondantes).

Enfin :

Un graphe est parfait, si et seulement si, il ne comporte ni trou impair ni antitrou impair : ceci est présumé vrai : ceci est la conjecture du graphe parfait de Berge. Ceci n'est pas un théorème!

Les petits énoncés ne font-ils pas de grandes conjectures ?

En mai dernier, sur son lit d'hôpital, Claude apprend qu'une preuve lourde, très lourde, confirmerait presque sûrement sa conjecture. La certitude adviendrait-elle avant juillet ? La publication de la preuve par Chudnovsky, Robertson, Robin et Seymour est annoncée aujourd'hui pour 2003. Confirmée ou infirmée une conjecture ne survit pas à sa preuve, elle devient théorème : ceci est un terrible événement.

Dans la photographie ci-contre, on voit Claude Berge montrer du doigt un vrai trou dans une de ses auvres en os à moelle.

\section{NAISSANCE DES HYPERGRAPHES}

Inventeur, innovateur, Claude Berge a poursuivi sans cesse une idée forte : théoriser les problèmes de réseaux considérés comme des configurations combinatoires. Empruntant au vocabulaire anglo-saxon, sa « théorie des graphes » contribue à renouveler le champ des mathématiques discrètes en multipliant les problèmes structuraux des configurations réticulées: les aspects topologiques et géométriques y prennent le pas sur les dénombrements d'objets finis qui étaient jusqu'alors la préoccupation centrale de la combinatoire. Il avait dix ans en 1936, année où sont publiés les théorèmes fondateurs de la théorie des graphes sous les noms de Birkhoff, Dehn, Erdös, Harmegnies, Heawood, König, Menger, Motzkin, Pólya, Wagner et Whitney : ce sont autant de guides qui vont le stimuler sa vie durant pour colmater une théorie féconde certes, mais très échevelée, qu'il généralise en théorie des hypergraphes. 
Dans sa Théorie générale des Jeux à $n$ personnes, c'est au moyen des graphes de positions de jeux qu'il définit stratégies et équilibres. Il invente alors le concept original d'équilibre fort : un joueur qui choisit sa stratégie dans un équilibre fort oblige tous les autres à faire de même. L'équilibre fort de Berge est stable par rapport à la déviation de tous les joueurs sauf l'un d'entre eux, alors que l'équilibre de Nash l'est par rapport à tous les joueurs; d'où son grand intérêt stratégique vu la multiplicité éventuelle des équilibres.

Dans le livre «Programmes, jeux et réseaux de transports », écrit avec Alain Ghouila-Houri, la recherche opérationnelle trouve ses fondements abstraits autour de la convexité, la séparation et la dualité vectorielle. L'étude des points-selles et des formules minimax conduit Claude Berge à généraliser en termes de polyèdres le théorème fameux de König et Hall, à savoir que dans tout graphe biparti, le plus petit cardinal d'un ensemble de sommets incident à toutes les arêtes est égal au plus grand cardinal d'un ensemble d'arêtes sans incidences communes.

Il définit les matrices équilibrées $A$, matrices zéro-un ne comportant pas de sousmatrice carrée d'ordre impair avec exactement deux 1 dans chaque ligne et chaque colonne. Sous cette condition, les deux polyèdres

$$
\left\{x \in \mathbb{R}^{\mathrm{n}}: A x \geq e, x \geq 0\right\} \quad \text { et } \quad\left\{y \in \mathbb{R}^{m}:{ }^{t} y A \leq e, y \geq 0\right\}
$$

ont tous leurs sommets à coordonnées entières. Et c'est en considérant les lignes des matrices $A$ comme des vecteurs d'incidence aux sommets-colonnes qu'il lance le vocable d'hypergraphe (sur une proposition spontanée de Jean-Marie Pla à une séance de séminaire de l'EHESS rue Richer). Claude Berge décline ensuite ses hypergraphes à l'instar des graphes en termes de cycles, de stabilité, de couplage, de coloration, d'isomorphisme, etc.

Ses manuels font recette et sont, pour certains, publiés en cinq langues. Une exception : un Que sais-je ? intitulé «La théorie des graphes » paru sous le n ${ }^{\circ} 1554$ aux Presses Universitaires de France en 1974, rédigé par les membres de notre séminaire commun à l'EHESS-MSH et signé Aimé Sache, a disparu à notre insu du catalogue.

\section{QUELQUES OUVRAGES À CONSULTER}

Nous proposons ci-dessous les références des travaux mathématiques principaux écrits ou dirigés par Claude Berge et accessibles en bibliothèque.

BERGE C., « Théorie générale des jeux à n personnes », Mémor. Sci. Math., n 138, Paris, Gauthier-Villars, 1957.

BERGE C., Théorie des graphes et ses applications, Paris, Dunod, Coll. Universitaire de Mathématiques, 1958.

BERGE C., Espaces topologiques : fonctions multivoques, Paris, Dunod, Coll. Universitaire de Mathématiques, 1959. English translation: Topological spaces. Including a treatment of multi-valued functions, vector spaces and convexity, New York, Macmillan, 1963; reprinted by Dover Publication, Inc., Mineola, NY, 1997. 
BERGE C., « Some classes of perfect graphs », Six papers on Graph Theory, Indian Statistical Institute, Calcutta, 1969, p. 1-21.

BERGE C., Principes de combinatoire, Paris, Dunod, 1968. English translation: «Principles of combinatorics », Mathematics in Science and Engineering, vol. 72, New York-London, Academic Press ,1971.

BERGE C., «Graphes et hypergraphes », Monographies Universitaires de Mathématiques $\mathrm{n}^{\circ}$ 37, Paris, Dunod, 1970. English translation: «Graphs and hypergraphs », vol. 6, Amsterdam-London, North-Holland Publishing Co.; New York, American Elsevier Publishing Co. Inc., 1973. Second revised version: Paris-BrusselsMontréal, Dunod, Coll. Dunod-Université, série Violette, $\mathrm{n}^{\circ}$ 604, 1973. English translation: vol. 6, Amsterdam-London, North-Holland Publishing Co.; New York, American Elsevier Publishing Co. Inc., 1976.

BERGE C., « Hypergraphs generalizing bipartite graphs », Integer and nonlinear programming, J. Abadie (ed.), Amsterdam, North-Holland, 1970, p. 507-509.

BERGE C., « Balanced matrices », Math. Programming 2, 1972, p. 19-31.

BERGE C., «Introduction à la théorie des hypergraphes », Séminaire de mathématiques supérieures $\mathrm{n}^{\circ}$ 51, Montréal, Les Presses de l'Université de Montréal, 1973.

BERGE C., Hypergraphes. Combinatoire des ensembles finis, Paris, Dunod 1987. English translation: Hypergraphs. Combinatorics of finite sets 45, Amsterdam, NorthHolland Publishing Co., North-Holland Mathematical Library, 1989.

BERGE C., CHVÁTAL V. (eds), «Topics on Perfect Graphs », North-Holland Mathematical Studies 88, Annals of Discrete Mathematics 21, Amsterdam-New York, North-Holland Publishing Co., 1984.

BERGE C., GHOUILA-HOURI A., Programmes, jeux et réseaux de transport, Paris, Dunod, 1962. English translation: Programming, games and transportation networks, London, Methuen and Co., Ltd.; New York, John Wiley and Sons, Inc., 1965.

Colloque Claude Berge, Centre International de Calcul, Rome, juillet 1966, Théorie des graphes, journées internationales d'étude, P. Rosenstiehl (dir. scientifique), Paris, Dunod, 1967.

Colloque Claude Berge, Orsay, 8-13 juillet 1976, Problèmes Combinatoires et Théorie des Graphes, Colloques internationaux du C.N.R.S., $\mathrm{n}^{\circ} 260$.

Colloque Claude Berge, Marseille-Luminy, juin 1990, «Graph Theory and Combinatorics », Discrete Mathematics, C. Berge, D. Bresson (guest editors), vol. 111, n 1-3, North-Holland, 1993.

Colloque Claude Berge, Marseille-Luminy, 5-12 septembre 1995, «Graphs and Combinatorics », Discrete Mathematics, J.-L. Fouquet, P. Rosenstiehl (guest editors), vol. 165-166, North-Holland, 1997.

Colloque Claude Berge, 2000, Bruce Reed (guest editor) [à paraître]. 
Pour finir cet éloge d'un ami artiste imprévisible, insaisissable, esprit furtif et parfois habile en malices, nous transcrivons le texte que nous lui avons confectionné (toujours pour les lectures des Jeudis de l'Oulipo) où phrase après phrase on finit par trouver les mots qui disent la fameuse conjecture des graphes parfaits.

\section{LA DÉCOUVERTE DE LA CONJECTURE DE BERGE PAR CLAUDE}

Un monde émergeant s'annonce merveilleux, ssi, il n'engendre ni trou encombrant ni verbe inconsistant.

Un couvent d'alchimistes se révèle puissant, ssi, il n'accepte ni moine trop pur ni antimoine impur.

Un brocanteur rural semble honnête, ssi, il ne colporte ni caille trouvée ni antiquaille recelée.

Une riche belle-mère se trouve refaite, ssi, elle ne détient ni dot gènique ni antidote générique.

Une chorégraphe distraite reste vierge, ssi, elle ne produit ni corps visible ni anticorps nuisible.

Un épigraphe sur vergé paraît surfait, ssi, il ne contient ni date impaire ni antidate mensongère.

Un graphe de Berge est parfait, ssi, il ne comporte ni trou impair ni antitrou impair.

Pierre ROSENSTIEHL 